\title{
The DECIDE Science Gateway
}

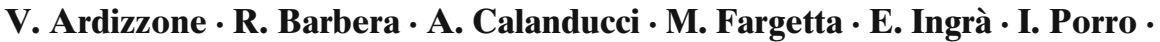 \\ G. La Rocca - S. Monforte · R. Ricceri • R. Rotondo • D. Scardaci • A. Schenone
}

Received: 14 December 2011 / Accepted: 2 October 2012 / Published online: 27 October 2012

(C) The Author(s) 2012. This article is published with open access at Springerlink.com

\begin{abstract}
The motivation of this work fits with the general vision to enable e-health for European citizens, irrespective of their social and financial status and their place of residence. Services to be provided include access to a high-quality early diagnostic and prognostic service for the Alzheimer Disease and other forms of dementia, based both on the European Research and Education Networks and the European Grid Infrastructure. The present paper reports on the architecture and
\end{abstract}

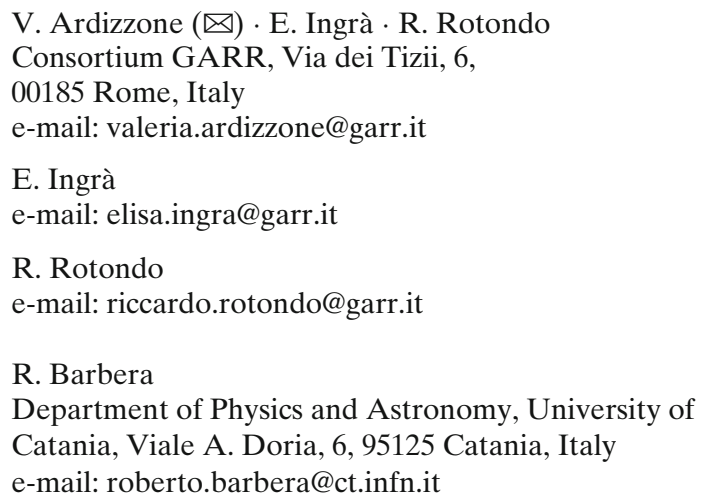

A. Calanducci - M. Fargetta

Consorzio COMETA, Via S. Sofia, 64,

95123 Catania, Italy
A. Calanducci
e-mail: antonio.calanducci@ct.infn.it
M. Fargetta
e-mail: marco.fargetta@ct.infn.it

services of a Science Gateway developed in the context of the DECIDE project, which aims to support the medical community in its daily duties of patients' examination and diagnosis. The implementation of the Science Gateway is described with particular focus on the standard technologies adopted to ease the access by non IT-.expert users. The work leverages on an authentication and authorization infrastructure based on Identity Federations and robot certificates and on the

\footnotetext{
I. Porro $\cdot$ A. Schenone

Department of Computer Science, Bioengineering, Robotics and Systems Engineering, University of Genoa, Viale Causa 13, 16145 Genoa, Italy

I. Porro

e-mail: ivan.porro@nextage-on.com

A. Schenone

e-mail: andrea.schenone@unige.it

G. La Rocca - S. Monforte · R. Ricceri - D. Scardaci

Division of Catania, Italian National Istitute of

Nuclear Physics, Via S. Sofia, 64, 95123

Catania, Italy

G. La Rocca

e-mail: giuseppe.larocca@ct.infn.it

S. Monforte

e-mail: salvatore.monforte@ct.infn.it

R. Ricceri

e-mail: rita.ricceri@ct.infn.it

D. Scardaci

e-mail: diego.scardaci@ct.infn.it
} 
adoption of the SAGA standard for middlewareindependent Grid interaction. The architecture and the functionalities of the digital repository for medical image storage and analysis are also presented.

Keywords e-health service - Grid computing • Science gateway $\cdot$ Standard-based development and middleware-independent deploy

\section{Introduction}

The field of medical imaging has developed enormously in the past 20 years. Image databases, made of thousands of medical images, are currently available to be used as a reference for individual diagnosis. At the same time, sophisticated and computationally intensive algorithms have been developed to extract information, invisible to the naked eye, from medical images. In particular, brain diseases are good candidates to benefit from such applications. Highly prevalent and burdensome chronic conditions, such as Alzheimer Disease (AD) and other neurodegenerative and neurodevelopmental disorders, can be early diagnosed by means of image- and signalbased markers of structural and functional brain changes, allowing early pharmacological or rehabilitative interventions. Each year, 1.4 million people in Europe develop a form of dementia (one every $24 \mathrm{~s}$ ) and it is believed that there are currently 7.3 million people in the continent living with dementia and about 35.6 million worldwide. This number is also estimated to nearly double over the next 20 years and to reach 65.7 million in 2030. In 2008, the total cost for illness of dementia disorders in the European Union was estimated to 160 billion Euro $56 \%$ of which were costs of informal care. Because of the ageing population, and the increasing pressures on public finances, dementia is deemed to become one of the major issues in the next decades for the sustainability of national health systems.

Unfortunately, neuroinformatics advancements require high computational and storage resources as well as large reference image datasets of normal patients, limiting its spread to advanced academic hospitals and research centres equipped with appropriate human expertise and hardware facilities.

The aim of the Diagnostic Enhancement of Confidence by an International Distributed Environment (DECIDE) project [1], co-funded by the European Union under its Seventh Framework Program, is to design, implement, and validate a dedicated e-Infrastructure based on the PanEuropean backbone GÉANT [2] and the National Research and Education Networks (NRENs) and relying on the European Grid Infrastructure (EGI) [3] and the National Grid Initiatives (NGIs). The DECIDE clinical infrastructure is also connected to the research infrastructure of the neuGRID [4] project.

Over this e-Infrastructure, a production quality service is provided around the clock for the computer-aided extraction of diagnostic disease markers for $\mathrm{AD}$ and schizophrenia. DECIDE offers access to a big distributed reference databases ( 850 and 2,200 datasets of normal and neurological subjects, respectively), large distributed computing and storage resources (more than 1,000 CPU cores and 70 TB of disk storage), and intensive image and signals processing tools.

DECIDE is focused on supporting neurologists and physicians involved in the assessment of neurodegenerative diseases in the diagnosis and prognosis and aims at enhancing user confidence by improving the reliability of the required analysis and by integrating different clinical approaches. It has been conceived to target a non-technical medical audience and aims to support the daily needs of neurologists while dealing with their patients, going well beyond the world of research.

Four applications have been deployed on the DECIDE infrastructure:

- Automated segmentation of hippocampal volume from structural magnetic resonance images to support the diagnosis of AD (GridMRISeg);

- Voxel-based statistical analysis of 18F-FDG positron emission tomography (PET) and Tc99-ECD single photon emission tomography (SPECT) to assess patterns of brain hypometabolism and hypo-perfusion to support the diagnosis of AD (GridSPM); 
- Spectral-based statistical analysis of electroencephalographic studies, used for the extraction of quantitative electrophysiological markers to support the diagnosis of AD (GridEEG);

- Pattern recognition analysis of functional 18FDOPA PET neuroimaging studies for the extraction of class-related biomarkers in the classification of schizophrenic patients (GridANN4ND).

Mainly, their goal is to provide doctors at peripheral hospitals with service tools for determining clinical markers for the early diagnosis of neurological and psychiatric disorders (neurodegenerative diseases and schizophrenia) together with their prognostic relevance.

DECIDE applications and tools are exposed to the end users (neurologists, physicians, and scientists in general) through a Science Gateway $[5,6]$.

In this paper, the architecture and current implementation of the DECIDE Science Gateway is presented from the point of view of the standard technologies adopted. The paper is organized as follows. Section 2 describes the architecture of the DECIDE Science Gateway and the methods and technologies used to build the applications portal. Separate sub-sections are devoted to the e-Infrastructure and the Grid Engine. Section 3 reports on the DECIDE Science Gateway services implemented so far and on the results already obtained. Conclusions are then drawn in Section 4.

\section{Methods}

\subsection{The Application Layer}

The DECIDE platform consists of three different layers: research networks, Grid resources and domain-specific applications.

Network connectivity, provided by the GÉANT backbone and the National Research and Education Networks of the countries participating in the project, brings together different types of structures (clinical and research centres and academic research institutions) interconnected among them by high speed and huge bandwidth links.
The Grid infrastructure is used as a platform to enable collaboration among all partners, as a technological "glue" to harmonize and unify developments, and as an elastic pool of computing and storage resources where large volumes of data can be hosted and related analyses can be performed.

The approach adopted by DECIDE regarding its applications ensures the requirements of the neurological community are taken into account from the very beginning in the design of application services to reach full usability in a real clinical environment. The use of different medical acquisition data (Magnetic Resonance Imaging - MRI, Positron Emission Tomography PET, and Electroencephalography - EEG) allows the combination of complementary diagnostic approaches on neurodegenerative disease diagnosis, enabling synergies between different clinical domains and possibly supporting correlation studies among different neurological approaches. DECIDE applications, as already mentioned in the previous section, implement four different diagnostic/prognostic algorithms comparing the neuroimaging and electrophysiological data of the suspect patients to those contained in a large reference database shared by the hospitals interconnected by the e-Infrastructure.

The four algorithms are shortly described below:

- GridSPM [7]: specifically designed for SPECT and PET neurological clinical images, provides a statistical analysis on a single-subject, based on Statistical Parametric Mapping (SPM) for the early diagnosis of Alzheimer Disease and other neurodegenerative diseases;

- GridANN4ND [8, 9]: concerns the analysis of PET biomarkers in Neurological and Psychiatric Disorders and provides a single-subject classification of suspected patients through the use of an Artificial Neural Network;

- GridMRISeg [10]: implements an automatic algorithm for the subcortical segmentation of single-subject MRI brain images for hippocampal volume estimation, using the auto context model (ACMAdaboost) developed by LONI [11]; 
- GridEEG [12-14]: based on a comparison of pathological versus normal subjects, implements EEG processing algorithms with the aim of detecting early symptoms of AD and distinguishing different forms of degenerative impairment.

Furthermore, the project aims to design and implement a multimodal repository, to include MRI, PET/SPECT and EEG datasets and make them available for exploitation by the data analysis software of the diagnostic/prognostic services. Medical data ownership remains in control of the physicians who contribute with their medical data to the medical repository, uploading data and reports according to their relevant authorization rights. By design, no free download of medical data from the DECIDE repository can be possible. Experts and scientists can only use the medical data contained inside the repository through the DECIDE diagnostic/prognostic services according to their authorisations.

Besides the configuration of applications, attention had therefore to be paid to the roles that users can play in accessing the DECIDE services, as well as to the authorization and permission policies enacted by the DECIDE portal. Four different kinds of users have been identified: neurologists, physicians, scientists and data managers each having different roles in the analysis process.

Neurologists are involved in the whole diagnostic and therapeutic process and are enabled to perform diagnosis by combining the response of a number of diagnostic tests. They have permissions to access a restricted number of DECIDE services. First, they can open a diagnostic session associated to a specific patient and upload his/her clinical data. They can select the requested applications for each patient and choose, from a list of authorized institutions, the one which will be in charge of running each requested application. They may eventually download the final diagnostic report.

Physicians are authorized to run one or more specific applications either within a standard diagnostic session opened by a neurologist or by creating a diagnostic session on their own. Within a session associated to their institution, they can run applications, without the right to set and/or modify the values of their parameters, and can finally validate the diagnostic report to be downloaded by the neurologist.

Scientists can access the advanced diagnostic sessions which their institution has been associated to by neurologists or open an experimental session for research purposes. Running the applications, they can, unlike physicians, modify the parameters values for the related algorithms and set up the configuration of final reports.

Data Managers can populate and update the DECIDE repositories, maintaining data and metadata, and train and update the artificial neural networks used by some applications.

A number of issues had to be tackled and solved in order to define a general architecture of the deployment of the DECIDE services on the Grid infrastructure. Two different types of services are available through the DECIDE portal:

- Front-end services, devoted to clinical and scientific users for running diagnostic services supporting neurologists in their diagnoses. Three front-end services are provided: the management of diagnostic sessions (used by neurologists), a basic application execution (used by physicians), and an advanced application execution (used by scientists).

- Back-end services, designed to be used by data managers for managing and maintaining reference databases, and for training artificial neural networks. Data managers for each application have different implementations of the same back-end service.

Aimed at supporting a clinical approach, the "diagnostic session service" was defined in order to integrate results from different analyses relative to the same patient and can be opened by neurologists and/or by physicians/scientists for each patient undergoing the diagnostic process. The "diagnostic session service" is configured to allow Neurologists, Physicians and Scientists to interact and collaborate during the diagnostic sequence. Data and metadata are shareable among them and they can exchange messages.

To access the DECIDE e-services, users have first to be registered on the DECIDE Science Gateway and receive the authorisation by the 
DECIDE Consortium upon the fulfillment of specific requisites. Once registered, users are then allowed to sign in. After a successful login, neurologists can open a new diagnostic session concerning a specific patient. They can choose between opening a standard session or an advanced one. The user is requested to fill a form with clinical data related to the patient, to flag one or more analyses to be executed and to associate a physician (standard session) or a scientist (advanced session) to each application he/she wants to be run according to the type of session. Actually, neurologists associate a department to the session and all physicians (scientists) belonging to this department can access the session.

Also physicians and scientists can open a new session and directly run the application(s) they are authorized to. All authorized physicians working at a department can view a list of all the standard sessions associated to their institution. Similarly, all authorized scientists working at a department can view the list of all the advanced sessions associated to their institution. Both physicians and scientists can select a session from the list and run for this session the application(s) they are authorized to.

The physician user can run an application, which he/she has the authorization for, within a standard diagnostic session, opened by a neurologist or during a diagnostic session he/she has opened on his/her own. The scientist user can run an application which he/she has the authorization for, either within an advanced diagnostic session opened by a neurologist or directly on his/her own for research purposes. Physicians or scientists can upload patient data files to be analysed through the application they are running. They must of course be aware of the requirements for input data of each application and should have performed a quality check on those data before uploading them.

Two different ways of running applications are possible. The physician user runs the DECIDE applications with a fixed set of values assigned both to parameters involved in algorithms and to configuration parameters for the diagnostic report. The scientist user is enabled to set up and modify a predefined number of parameters concerning algorithms, as well as some parameters defining the layout and the content of the diagnostic report.

All users (neurologists, physicians and scientists) can download the final diagnostic reports output by the applications.

The Catania Grid Engine described in Section 2.3.4 implements the above described applications and interacts with the DECIDE infrastructure through the Science Gateway. User interfaces for applications and diagnostic sessions are exposed to end users through standard components. Grid transactions are secured by proxies created by robot certificates (see below) while data management services are called through REST functions.

The DECIDE back-end services are designed mainly with the aim of providing tools for the maintenance of data and algorithms used by applications. Through these services, back-end users (data managers and developers) can either access reference databases or upgrade algorithms related to different applications.

The data manager user is provided with tools for adding and removing data concerning reference subjects and for updating the related metadata. The user can access only the specific data in the reference database which he/she owns and is responsible for. Data in the databases are shared just for statistical purposes and can be accessed by the applications during their execution on the Grid worker nodes.

Data managers can also run or update the training of the artificial neural networks used in GridANN4ND and in GridMRISeg. They can of course update only the specific application(s) they responsible for.

\subsection{Requirements and Related Research}

As anticipated in the previous sections, DECIDE services are exposed to users through a Science Gateway. According to $[15,16]$, a Science Gateway is a "community-development set of tools, applications, and data that is integrated via a portal or a suite of applications, usually in a graphical user interface, that is further customized to meet the needs of a specific community". Science Gateways are playing an important role in scientific research performed using e-Infrastructures and their relevance will further increase with the 
development of more sophisticated user interfaces and easier access mechanisms. Through the highly collaborative environment of a Science Gateway, users spread around the world and belonging to various Virtual Research Communities can easily cooperate to reach common goals and exploit all the resources of the cyber-infrastructure they are entitled to use.

In the last years, several frameworks have been created to implement Science Gateways, such as GENIUS [17], P-GRADE [18] and the Vine Toolkit [19]. In DECIDE, in view of the long term sustainability of the developments, the requirement was to have a Science Gateway based on standards, capable of running with little or no modifications on e-Infrastructures having several middleware and allowing different views according to the different roles and privileges of the various users (see previous section). Furthermore, since the DECIDE service is made of sites belonging to the European Grid Infrastructure, it was mandatory to build a Science Gateway respecting the EGI VO Portal Policy [20] and the EGI Grid Security Traceability and Logging Policy [21]. The result of a comparative analysis of the existing solutions, conducted at the beginning of the project, was that no framework could have met all the requirement without a substantial investment in code analysis and extension. So, profiting of the many-years "in house" experience with the GENIUS portal, and of the parallel development of Science Gateways in the context of other EU funded projects, such as CHAIN [22], EUMEDGRID-Support [23], GISELA [24], and INDICATE [25], we decided to build a new framework.

The reference model adopted is sketched in Fig. 1.

Users belonging to different organisations and having difference roles and privileges within the community the Science Gateway is developed for can access different applications and run them in a seamless way on e-Infrastructures where different middleware might be deployed. The details of the implementation and the current status of the DECIDE Science Gateway are given in the following sub-section.

\subsection{The DECIDE Science Gateway}

This section describes the architecture and present status of the DECIDE Science Gateway. The Science Gateway is built within the Liferay web portal framework and portlet container [26] and it is fully compliant with the JSR 286 ("portlet 2.0") standard [27]. Liferay is currently the most used framework to build Science Gateways in the "Grid world" and ships with more than sixty portlets that can be easily combined (mashed-up) to build complex and appealing e-collaboration environments. Other $200+$ portlets are available in the repository of the Liferay community.
Fig. 1 The reference model of the DECIDE Science Gateway

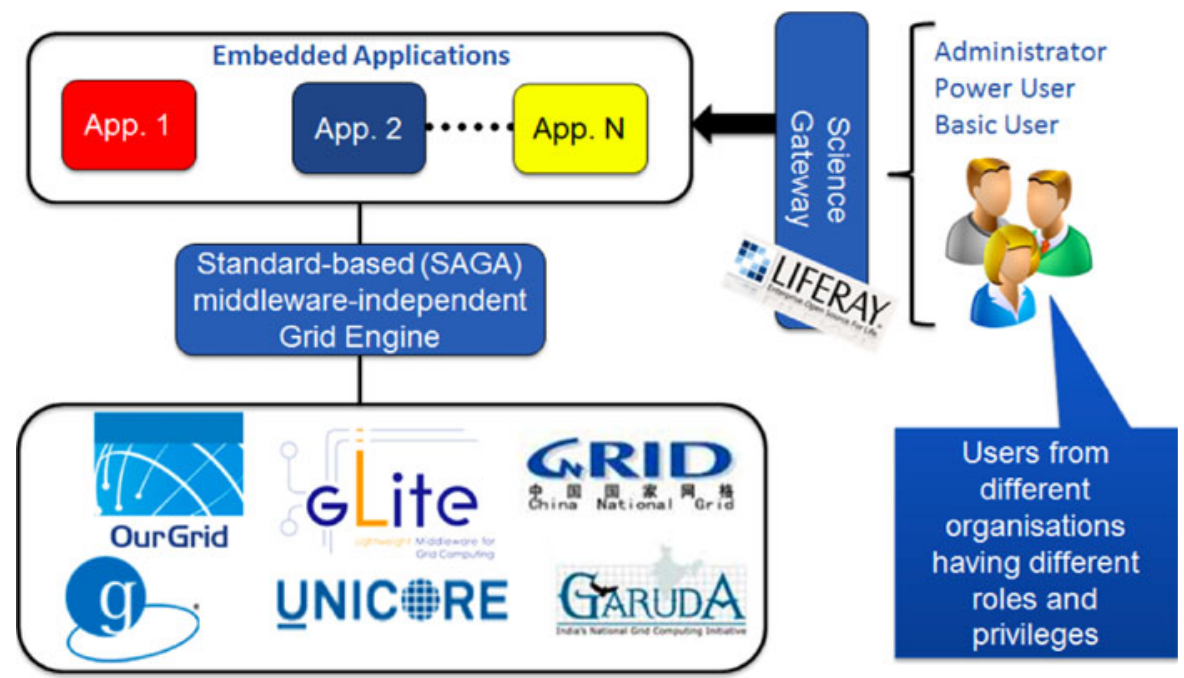


Separate sub-sections are devoted to the various functional aspects of the portal.

\subsubsection{Authentication and Authorisation}

The most important requirement of the DECIDE Science Gateway was to ease the access to the distributed computing and storage resources by the largest possible community of (non ITexpert) clinicians through a set of well-defined and domain specific applications. In order to meet this requirement, authentication and authorisation mechanisms have been conceived to provide a smooth access to the applications yet preserving the security level requested by the distributed eInfrastructure and the typology of the sensible information (clinical data) managed. Indeed, the neurological data stored in the Science Gateway have extra requirements in terms of security, anonymity and confidentiality. It must always be clearly defined who can access which images for his/her own analysis.

Therefore, several web and Grid technologies have been adopted and deployed to ensure that the authentication and authorisation mechanisms fulfill the stringent requirements and implement the expected roles and the corresponding privileges. Such tools implement an authentication/authorisation hierarchy moving from the web to the physical Grid resources in order for users to have to interact only with the top level tool, this taking care of transferring the credentials to lower levels.

The highest component in the authorisation/authentication hierarchy has to be integrated in the Science Gateway and has to support a Single Sign On (SSO) mechanism across all services a given user is entitled (i.e., has the right) to use, in order not to confuse non-experienced users with different sets of credentials.

Many web tools support SSO within a centralised or distributed authentication framework. Nevertheless, in order to comply with currently adopted standards and support the most relevant Identity Federations in Education and Research, the DECIDE Science Gateaway is compliant with the Security Assertion Markup Language (SAML) OASIS standard [28] for credentials communication. The Shibboleth [29] implementation of
SAML has been adopted in the DECIDE Science Gateway and a library has been developed to make Liferay manage the Shibboleth token.

In many countries there is currently a big effort to create Identity Federations gathering all education and research institutions to simplify and ease the access to services for users working in different locations. Actually, they are generally managed by National Research and Education Networks in EU countries and aim at the integration of networks, services and users. Therefore, it was important for DECIDE to follow this trend in order to allow its integration with other services and increase the number of potential users.

The use of Shibboleth allows an easy integration with Identity Federations (IdFs) and individual institutions (i.e., Identity Providers - IdPs) wishing to include the DECIDE Science Gateway as one of the resources (i.e., Service Providers - SPs) for their users. When a user tries to use one of the DECIDE applications available on the Science Gateway, he/she is re-directed to a Discovery Service (DS) listing all the supported IdFs and Identity Providers (IdPs) among which he/she can select the one he/she is member of. The IdP identifies the user, generally through a pair of username and password. If the authentication by the IdP is successful, the control is returned to the Science Gateway where user authorization is checked.

The DECIDE Science Gateway is a Service Provider of the GrIDP (Grid IDentity Pool) [30], a "catch-all" federation operated by INFN Catania and Consorzio COMETA to manage several Science Gateways and generic web portals. This is the federation collecting the IdPs of institutions which are not members of any Identity Federation and currently includes INFN (the Italian Institute for Nuclear Physics) and maat-g (a private company partner of the DECIDE consortium) IdPs. Besides GrIPD, the DECIDE Science Gateway is a SP of both the Italian official identity federation IDEM [31], one of the biggest Shibboleth-based federations available, and of the eduGAIN interfederation [32] gathering the many-million members of the IdFs of Belgium, Brazil, Croatia, Czech Republic, Finland, Germany, Greece, Hungary, Italy, Norway, Spain, Sweden, Switzerland, and The Netherlands. 
Once a user is authenticated, the authorisation system verifies his/her credentials. The Scientific Board of DECIDE grants authorisations and a centralised LDAP-based registry, connected to the DECIDE Science Gateway, has been created to store and manage roles and privileges. User roles are then mapped onto those performing Grid transactions using the Virtual Organisation Membership Service (VOMS) functionalities. The VOMS-based Virtual Organization vo.eudecide.eu has been created and four Groups have been defined, one for each application. Additionally, for each group, four Roles have been defined that represent the four different kinds of users of the DECIDE services explained in the.

A summary of the Groups and Roles available in the DECIDE Virtual Organization is shown in Fig. 2.

Besides the few developers of DECIDE applications, who need low-level access, the DECIDE VOMS is populated only by robot certificates who are used to sign Grid transactions with their proxies. Robot certificates and their use in the DECIDE Science Gateway are described in the next sub-section.

\subsubsection{Robot Certificates}

The management of personal certificates to access e-Infrastructures has demonstrated to be difficult by non-expert users and represents a limiting factor to the rapid spread of this technology in new scientific domains where computer science is not a basic knowledge. A notable step forward to make the access to Grid infrastructures as much transparent and smooth as possible has recently been achieved with the introduction of robot certificates,also referred as portal certificates, and their integration inside traditional general-purpose portals [17] and Science Gateways [33]. The advantages introduced by this new kind of digital certificates are manifold and ten Certification Authorities in Europe have already adopted them. For security sake, robot certificates are usually stored on board of tamper-resistant devices such as smartcards. This improves the security and avoids any fraudulent use of the private keys. A multi-threaded server, called eToken server, has been created and configured to manage a list of robot certificates (one certificate per application) stored in different USB

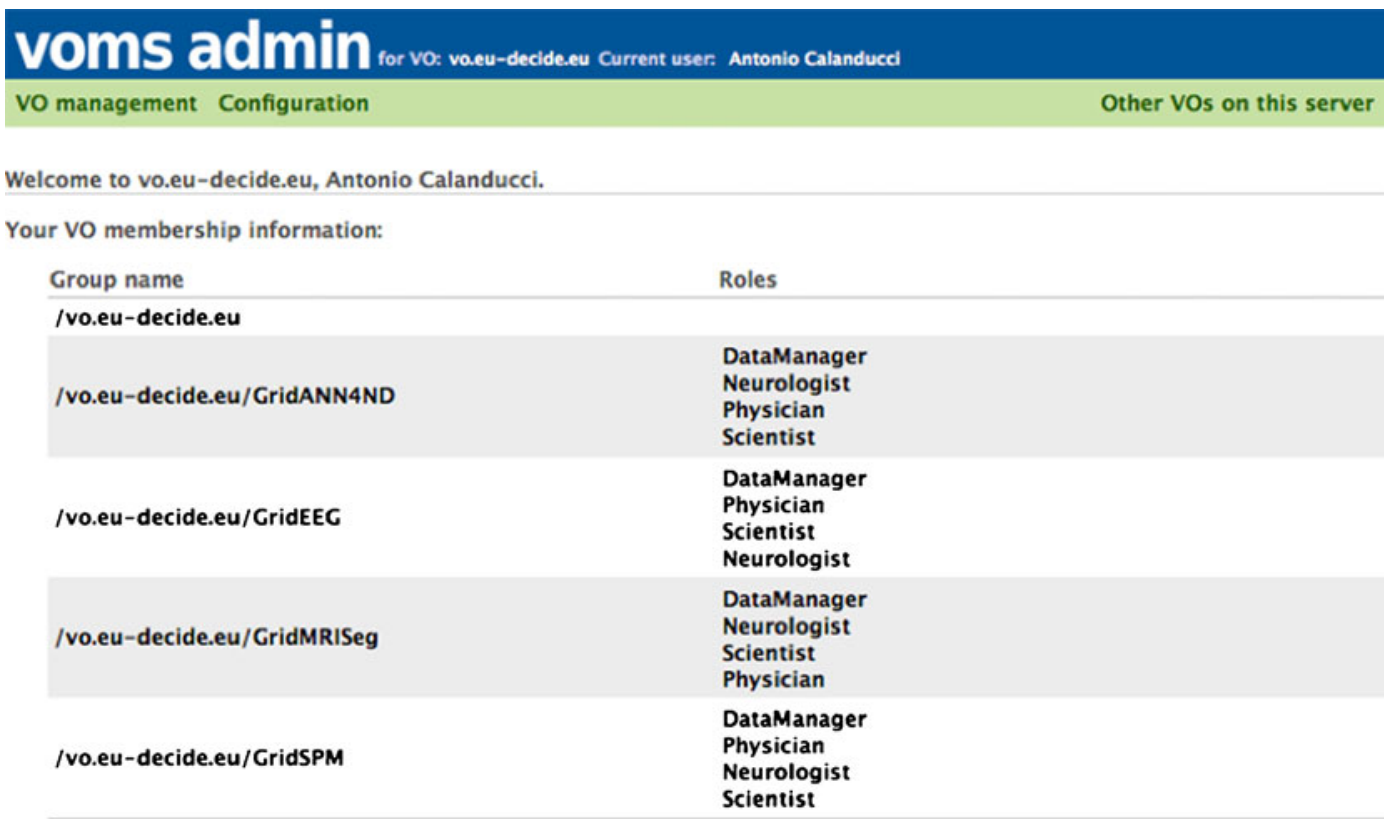

Fig. 2 The DECIDE Virtual Organisation Membership Service and its groups and roles 
eToken PRO 32/64 KB smart cards released by SafeNet [28]. The eToken Server provides the DECIDE Science Gateway (and other gateways developed at INFN Catania) with a $24 \times 7$ service and holds the web services to access the smart cards and interact both with the Virtual Organisation Membership Service and the automatic proxy renewal (MyProxy) service. This crypto library developed [33] combines different standards and programming native libraries with the latest release of robot certificates. A Java multi-platform client, configured for inter-service communication via HTTPS, completes the architecture. The eToken server is built on top of the Apache Tomcat Application Server and configured to accept requests only from a set of authorized "clients" (i.e., the Science Gateways). This ensures scalability and high performances especially when the server has to deal with huge numbers of requests. To further improve its performances and reduce the waiting time to get a proxy, the eToken Server implements also a cache mechanism.

The usage workflow of the "light-weight" Grid crypto library used by the DECIDE Science Gateway is shown in Fig. 3.

Once the accredited IdP has successfully authenticated the user, his/her authorisation rights on the local LDAP registry are checked and, if the verification is successful, he/she is logged into the DECIDE Science Gateway. Then, according to the information stored in the LDAP registry and the application(s) he/she wants to run from within the portal, the Science Gateway sends a requestID to the eToken Server. If the Science Gateway is authorised, and taking into account the information available in the HashMap, the eToken Server sends back to the Science Gateway a new proxy certificate, if the requestID is not found or the lifetime of the old proxy is expired. Otherwise it sends a cached proxy, if a valid proxy certificate is available in memory. This operation is completely transparent from the end-user point of view, and allows the user to execute the operations requiring the usage of the Grid services and get the results. The retrieval of a cached proxy takes only $20 \mathrm{~ms}$ while the creation of a new one requires about $5 \mathrm{~s}$.

\subsubsection{The Repository Manager}

One of the requirements of the DECIDE project is the creation and access of "reference databases" containing structured data related to normal patients' images. Those databases needed to be recreated in the adopted infrastructure, populated

Fig. 3 The workflow for implementing Grid authorisation by means of the eToken Server

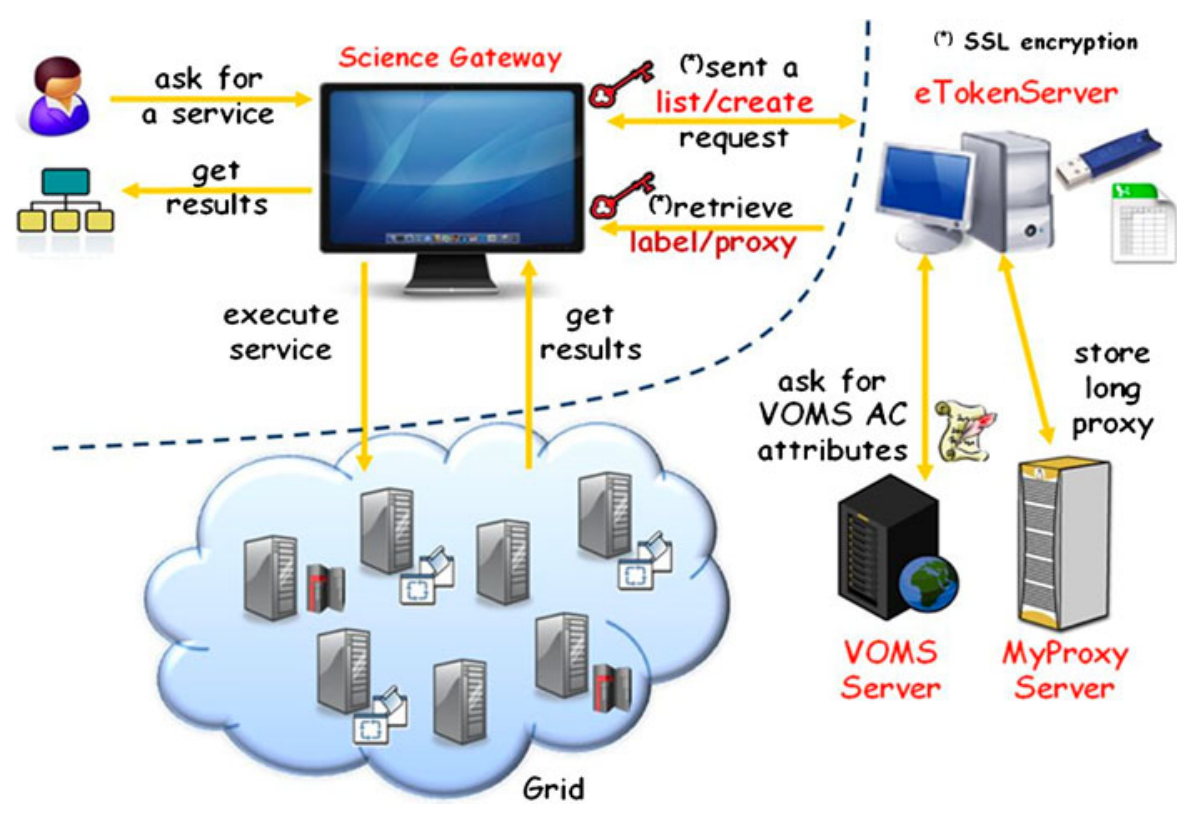


and later queried and accessed by other applications running in the worker nodes of Grid sites. In particular, four databases have been created and deployed on the DECIDE Grid infrastructure for each of the following types of data:

- PET/SPECT;

- EEG;

- MRI;

- PET/SPECT, to serve the GridANN4ND application.

Image files of normal patients need to be stored on the distributed storage resources of the infrastructure in an encrypted format. The aforementioned reference databases actually represent the metadata of those binary image files. For the sake of simplicity, we prefer to use the term "digital asset" to refer to the entity made of a binary image file and its set of metadata, and so we use the term "digital repository" (or simply "repository) to indicate a set of digital assets.

Four repositories have been created on the DECIDE e-Infrastructure, with the same names of the four reference databases. In order to achieve this, we have used the gLibrary framework [34] to create, access and manage digital assets on Grid. gLibrary, in fact, presents to users and applications the abstraction of digital asset as a unique entity, handling "behind the scenes" transactional interactions with the AMGA metadata catalogue [35] for managing relational metadata and to the Grid Storage Elements for managing binary files.

Data Managers of DECIDE need to access the repositories to add assets, by uploading anonymised patient data in an encrypted format and adding metadata from the reference database to those files. As those users are supposed not to be Grid experts, and being command line access considered forbidded by requirement, we developed an easy-to-use graphical interface in the form of a set of portlets integrated in the DECIDE Science Gateway. These portlets provide access both to the Secure Storage System $[36,37]$, used to encrypt the uploaded files and save the encryption keys into a keystore service located within the boundaries of hospitals, and to the gLibrary server that registers those files as assets and allows the addition and editing of their metadata. The interactions between the Liferay portlets and those two services are managed through their REST-based front-ends. To this goal, we have developed a set of APIs to perform all the available operations: encryption and replication of files to random Storage Elements of the infrastructure, addition and editing of their metadata.

The authentication of the APIs is done through the same Shibboleth-based mechanism used to access the DECIDE Science Gateway so a user trying to use the API without a valid Shibboleth token in his/her client (i.e., the web browser) would be refused.

For the sake of completeness, the steps and the interactions among the service to achieve the upload of an asset are explained below:

1. A user successfully logged in the Liferaybased Science Gateway retrieve the DECIDE Repository Manager portlet in his/her browser;

2. The user selects one of more files from his/her local filesystem (batch and asynchronous uploads are supported too) and transfer them via HTTPS to the "Uploader service" deployed within the boundary of the hospital he belongs too. One strong requirement of the service is that patient images should never cross the boundaries of the hospital that owns them in an unencrypted format.

3. The encrypt command is issued to the REST API of the Science Gateway. This API will act as a proxy to the machine providing the actual service, because of the Cross Origin Resource Sharing [37] problem that denies access to resources outside of the domain from which the original web content (the portlet in our case) has been retrieved;

4. The encrypted request is forwarded back to the REST API of the "Uploader Service", the service in charge of encryption and upload to the Grid storage resources;

5. The "Uploader Service" ask for and download a proxy certificate, according to the rights of the logged user (DataManager Role in our case) issued by the eToken Server 
(myproxy.ct.infn.it) needed for the subsequent Grid interactions;

6. The "Uploader Service" starts the encrypted upload, storing the encryption key into the keystore service (deployed in the same keystore.ct.infn.it machine), and moving the encrypted file to the first random storage element of the e-Infrastructure; notice that the files moved outside the hospital are transferred encrypted over the GSIFTP protocol;

7. A Logical File Name is assigned to the encrypted file and saved into the LCG File Catalog (LFC) that will keep track of this and his replicas;

8. At the end of the upload process, the client issues a "Replicate" command to the REST API of the Science Gateway;

9. 10. 11. In a similar way, the Replication request is forwarded to the REST API of the Uploader Service that in turn replicates the current uploaded file to a second random storage element and keep note of the destination on the Logical File Catalog service.

12. Once both the encrypted upload and replication phases are completed (Fig. 4), the user can fill all the metadata related to the uploaded file. All these requests are issued against the REST interface of the Science Gateway.

13. In turns, the Science Gateway forwards the metadata management requests to the gLibrary REST APIs.

14. gLibrary retrieves a proxy certificate from the eTokenServer according to the authorization of the logged user

15. On behalf of the user, the gLibrary service executes the proper sequences of commands towards the AMGA Metadata Service of the DECIDE Grid infrastructure.

\subsubsection{The Catania Grid Engine}

The DECIDE Science Gateway uses the Catania Grid Engine to perform Grid transactions. The Catania Grid Engine is a generic software module able to interconnect the Scientific Gateway presentation layer with the underlying Grid infrastructures using standard technologies. It allows the quick creation of new Science Gateways providing their developers with a simple interface and avoiding worry about middleware specificities.

In the design of the DECIDE Science Gateway, the adoption of international standards has been considered since the beginning as a mandatory practice in order to protect the investment in
Fig. 4 File encryption, uploading and replication with the Repository Manager

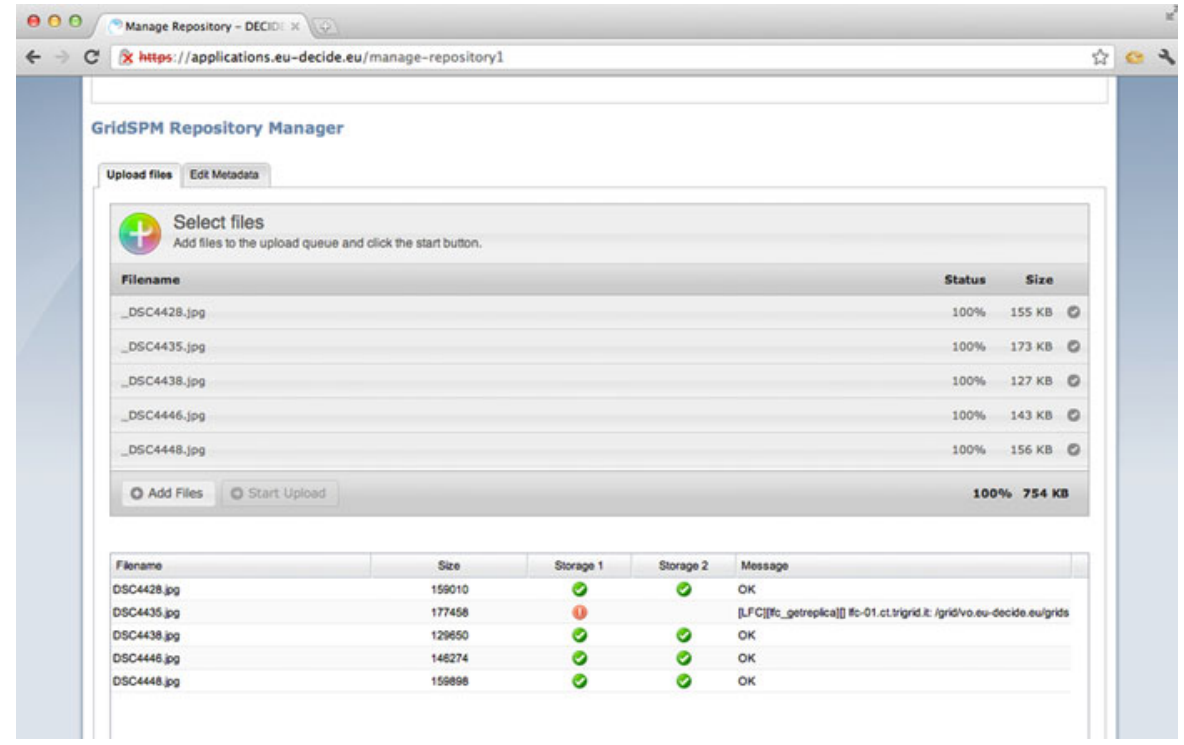


Fig. 5 The architecture of the Catania Grid Engine

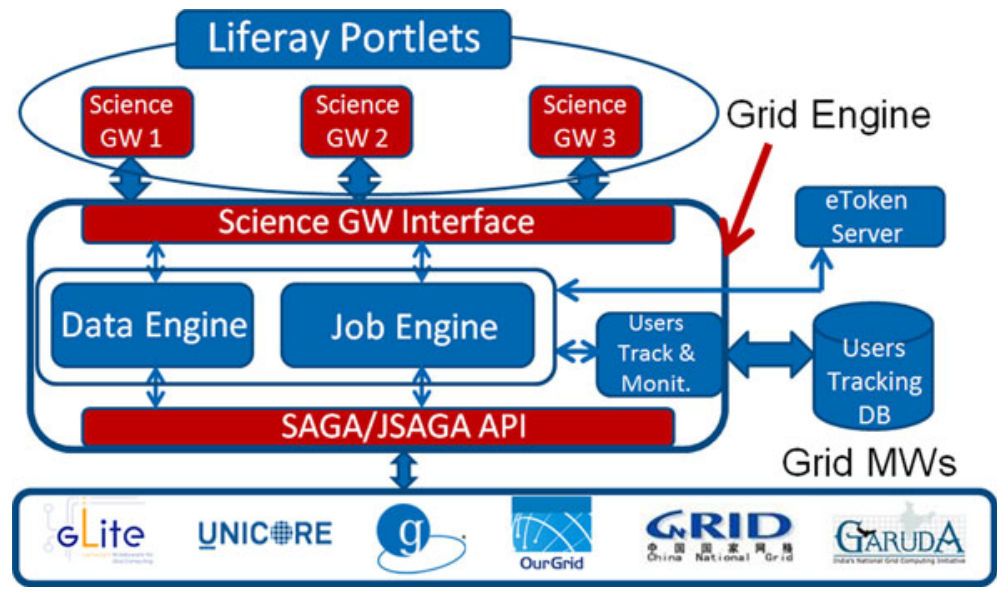

the creation of this high-level user interface from middleware changes and lack of interoperability. In this way, the Scientific Gateway can become a "gate" to a huge e-Infrastructure made of resources coming from different kind of Grid infrastructures with different middleware deployed and connected via standard interfaces. As a consequence of the above considerations, the Catania Grid Engine adopts the Simple API for Grid Applications (SAGA) Core API [38, 39], a high level, application-oriented, software library for Grid applications specified by the Open Grid Forum (OGF), and its JSAGA implementation [40]. JSAGA allows creating a unique interface to different middleware stacks and makes Science Gateways able to exploit resources coming from different Grid infrastructures.
The architecture of the Catania Grid Engine is sketched in Fig. 5.

The following modules are part of the Catania Grid Engine:

- The Science Gateway Interface: an interface hiding all the complexity of the Grid world to the presentation layer providing additional capabilities suitably designed for the web world. It allows developers to create portlets for applications in a very short time (just a few days).

- The Job Engine: a module allowing users to fully exploit Grid job management services. It maps job operations to JSAGA functions;

- The Data Engine: a module allowing users to fully exploit Grid data management services. It maps data operations to JSAGA functions;

Fig. 6 Thread Pool usage in the Job Engine

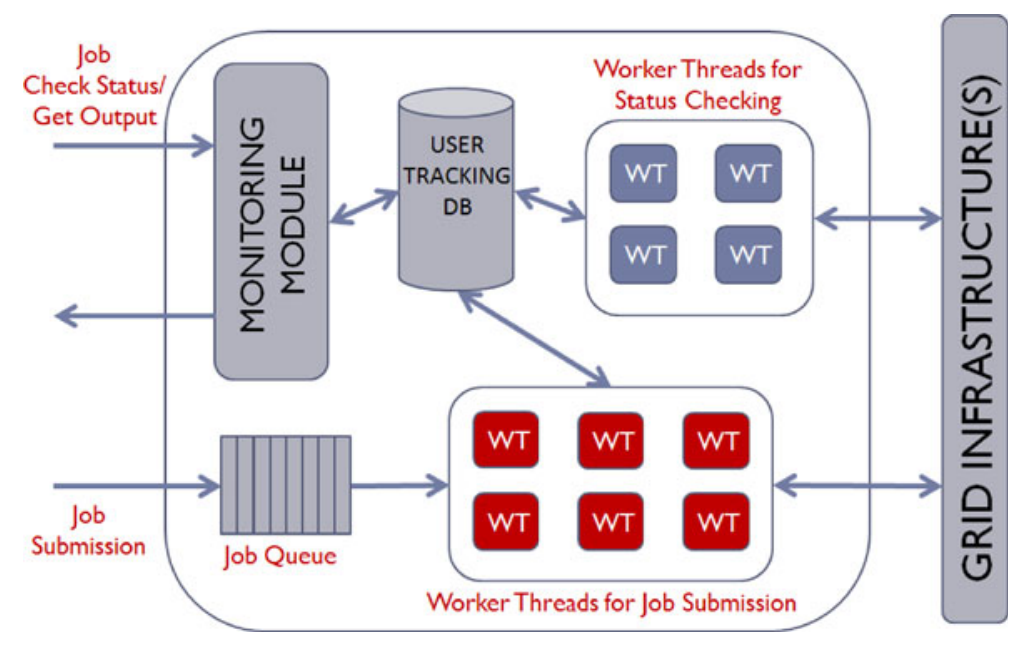


- The User Tracking and Monitoring DB: a user tracking and accounting tool able to store information on each Grid transaction in the Users Tracking database. It is in charge of controlling the rate of Grid interactions (in total and per users) executed via the Science Gateway to implement flow control policies (e.g., max number of jobs submitted per users, max number of jobs submitted perScience Gateway, etc.). It has been developed to make the DECIDE Science Gateway with the EGI VO Portal Policy [20] and the EGI Grid Security Traceability and Logging Policy [21].

\section{- The JSAGA API.}

In the rest of the sub-section we will concentrate our attention to describe the Job Engine which is the most relevant part of the Catania Grid Engine for what concerns the DECIDE Science Gateway. Readers interested in the Data Engine are forwarded to [41] for more information.

The Job Engine manages the whole cycle of job execution starting from the submission until the retrieval of the output. It receives requests to submit jobs from the Science Gateway interface and takes care of jobs until they are properly executed. Job operations such as submission, status check and output retrieval are mapped onto the functions of JSAGA. Moreover, the Job Engine deals with all the preliminary operations needed to execute a job such as associating a proxy to the job, looking for a working resource manager, satisfying special user requirements, and so on.

The Job Engine has an interface to the eToken Server to create proxies from robot certificates to be associated to a job. It also offers a fault tolerance capability by submitting a job to the Grid infrastructure until it will be properly executed shielding users from infrastructure failures.

Finally, it provides a User Tracking and Monitoring module with all the input information needed to account users operations and to control the Grid interactions rate.

In order to scale to large numbers of concurrent jobs and users (see next sub-section), the Job Engine makes use of the Java Thread Pools made available by the application server (Glassfish, in our case) where Liferay is running on (see Fig. 6).
Submitted jobs are queued and dispatched at constant rate as parallel threads of a pool so eliminating the risk that a large number of jobs can block the Science Gateway and induce a Denial of Service. The same concept of threads is used for job monitoring avoiding the Science Gateway to slow down when the status of many jobs is checked by users.

\section{Results}

As described in the previous sections, DECIDE is aimed at using e-Infrastructures to provide medical experts with a production quality service, operational around the clock, allowing doctors to execute algorithms on data coming from different diagnostic instruments in order to determine brain markers for the early diagnosis of $\mathrm{AD}$ and other forms of dementia (see Fig. 7).

This section describes the results obtained so far using the Science Gateway services.

\subsection{Diagnostic Sessions and Access to Applications}

The DECIDE front-end services are designed to create a multi-modal collaborative environment within the DECIDE platform. Different types of users may have the authorisation to access the DECIDE services, each having different permissions to services and data. In order to describe the results concerning the management of diagnostic sessions and the running of applications, two different scenarios have been tested as representative of users' behaviour in accessing the DECIDE Science Gateway.

Scenario 1: A neurologist managing a diagnostic session

A neurologist from a small hospital can access, without any training, the DECIDE services after registering to the DECIDE Science Gateway and being enrolled as an authorized user by the DECIDE Scientific Board. First, he/she has to open a diagnostic session related to a patient he/she wants to diagnose (see Fig. 8). To this goal, he/she needs to fill in the name of the patient as well as age and sex, choose the level of the session 
Fig. 7 DECIDE infrastructure and services view

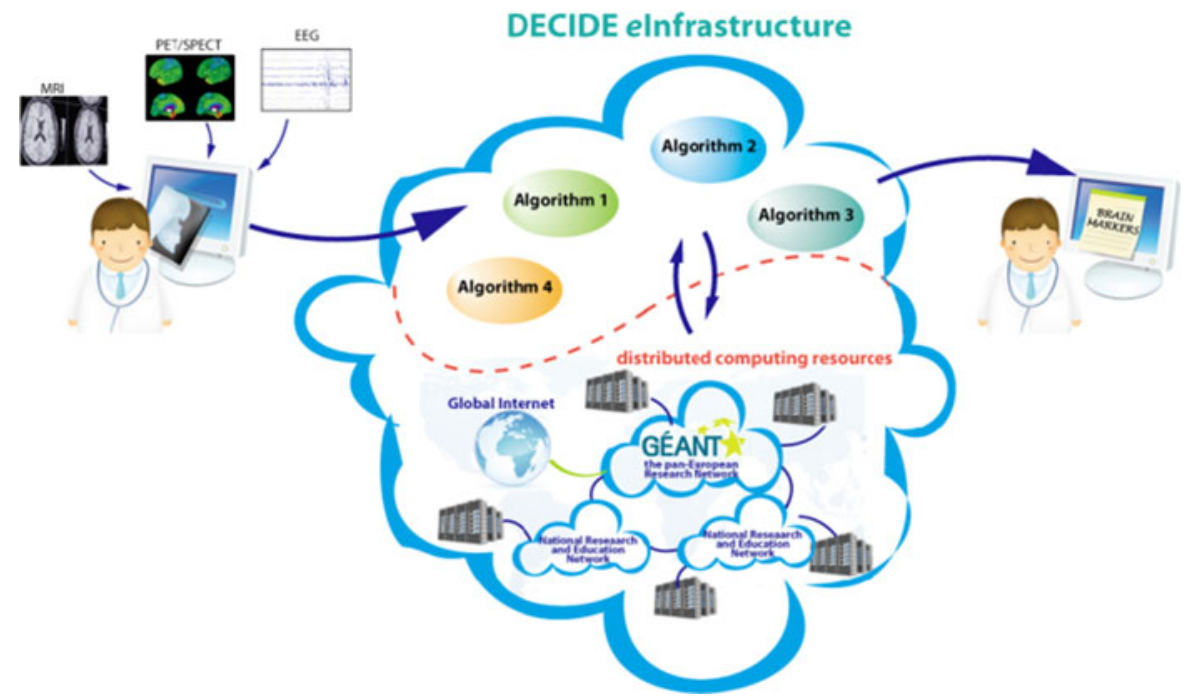

(basic or advanced), and flag one or more of the available DECIDE applications. When an application is selected, a drop-down menu appears with a list of departments with skilled clinicians for the selected level of that application. The neurologist has then to select the department he/she prefers (the closest to his/her hospital, a department he/she knows for professional reasons, etc.) and then he/she is enabled to start the session. The session is given a unique identifier by the system and is set to the status "open". Afterwards, the session is listed in the session list of the selected departments for each application and can be seen only by the doctors enabled to take it in charge. After an application has been run and completed, its status changes in the neurologist list of sessions and he/she is thus enabled to download the produced report.

Scenario 2: A physician running an application

A physician, having been trained to use one application, accesses the list of diagnostic sessions opened for the application he/she has been trained for and made visible to all the trained physicians
Fig. 8 Opening a diagnostic session on the DECIDE Science Gateway

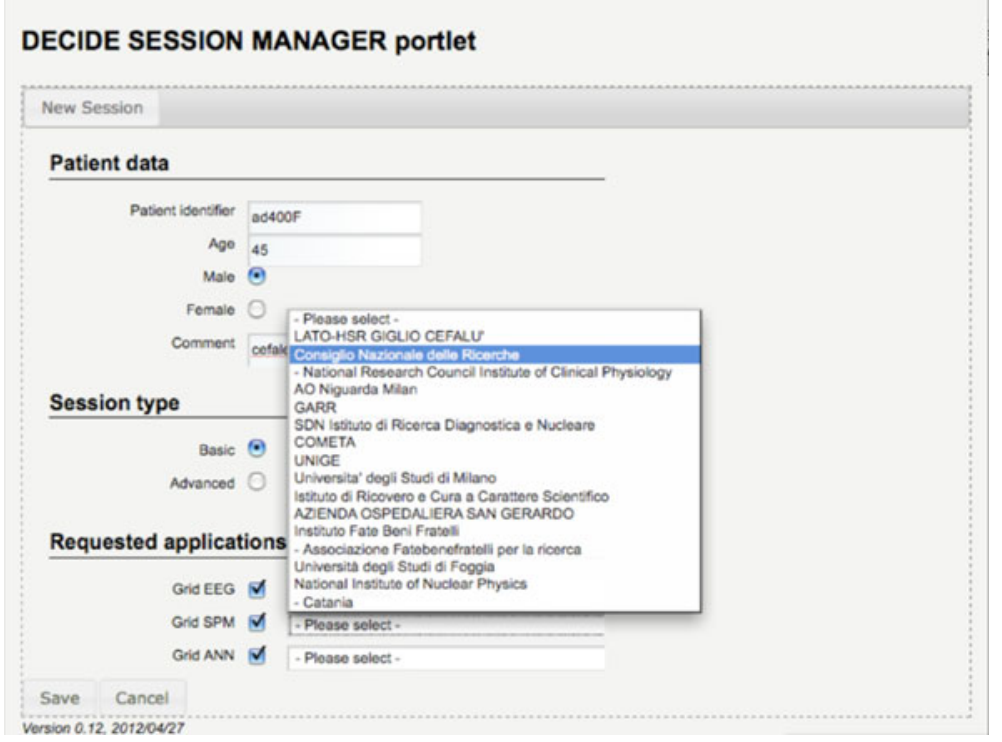


Fig. 9 Input page of the GridSPM application available on the DECIDE Science Gateway

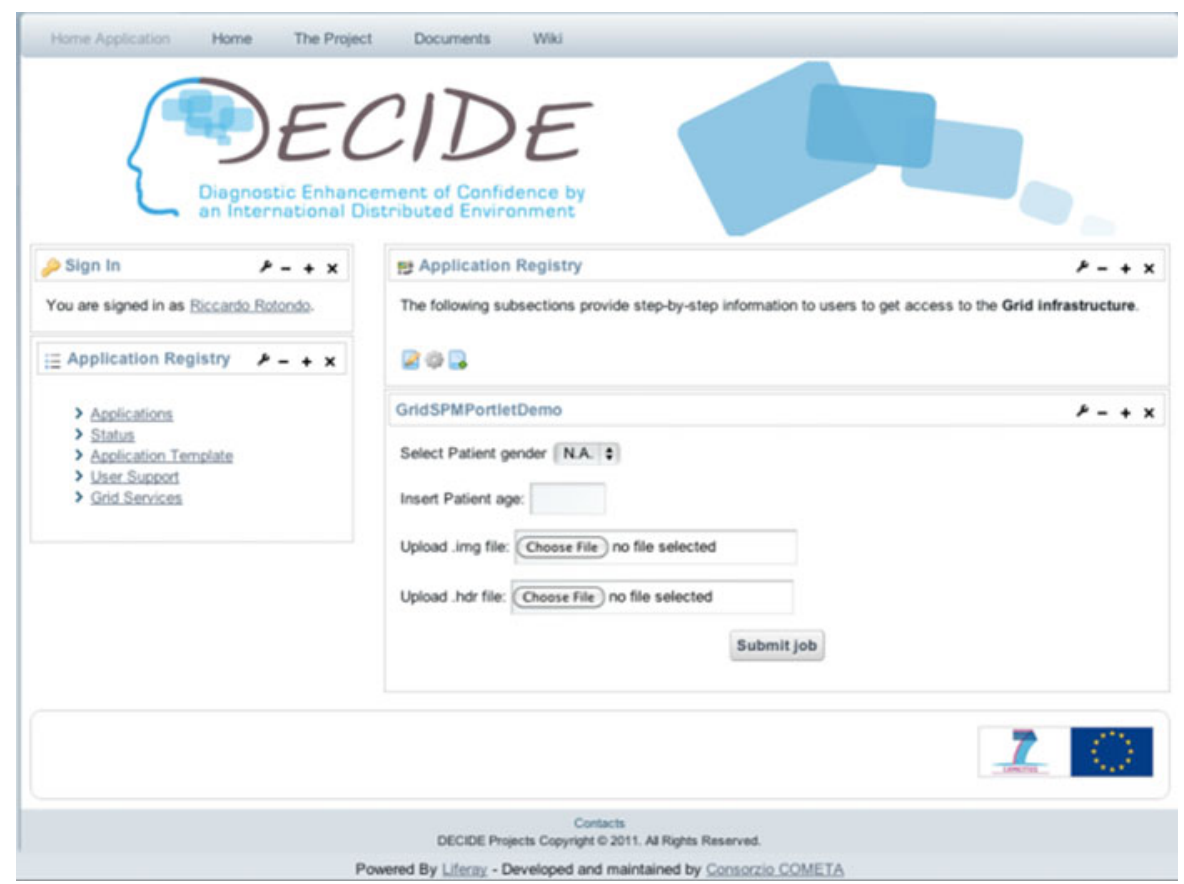

of his/her department. He/she can select a session associated to a patient whom he/she is in charge of and start running the related application. A user interface enables him/her to upload the input data and to fill in the metadata needed for the application through an interactive form. After having provided the requested data, he/she can run the application and go back to the list of diagnos- tic sessions. The execution of the application is monitored by the Science Gateway, through the Job Engine described above, and when it is over, a diagnostic report is produced he/she is warned about its availability. Then, he/she can download and check the report in order to validate it. If the report is approved, he/she may flag his/her application associated to that session as "done".
Fig. 10 Metadata insertion

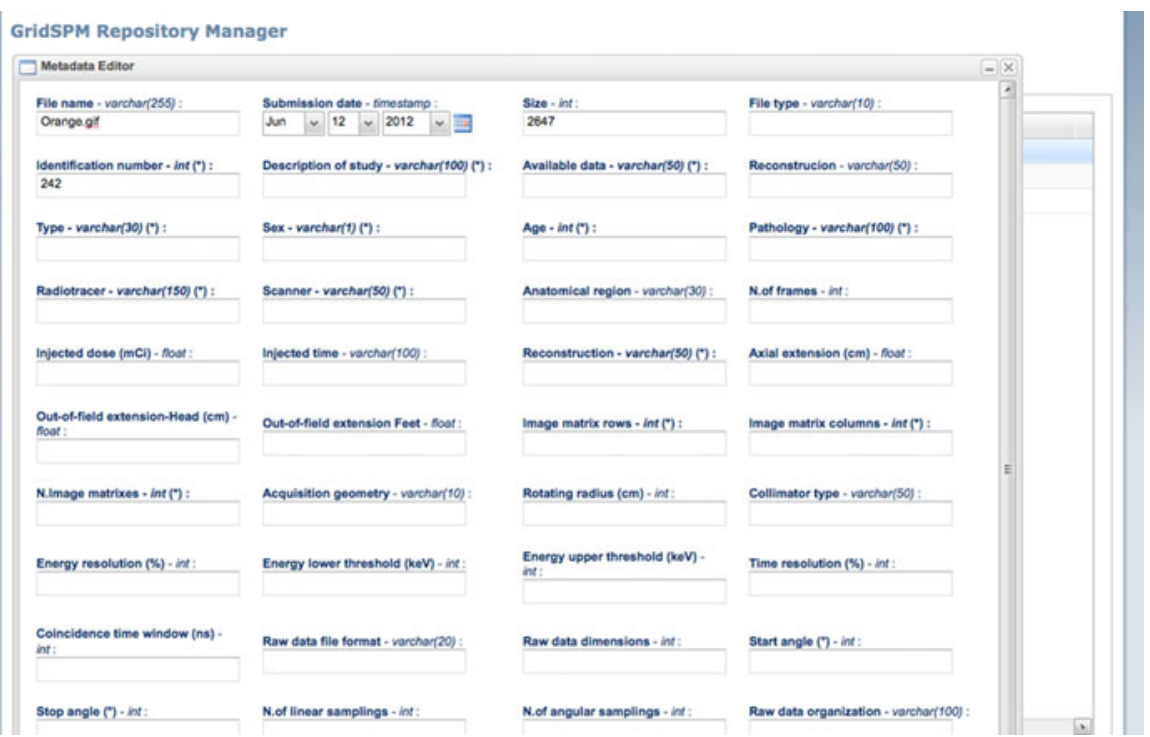


When an application associated to a diagnostic session has been completed, a warning is sent to the neurologist owning the session and he/she can see the application as "completed" in the list of his/her sessions. Finally, he/she is enabled to download the diagnostic report validated by the physician.

As an example, Fig. 9 shows the input page of the GridSPM application available on the DECIDE Science Gateway. To submit a GridSPM job, users just have to select the patient gender, insert the patient age, select the input images, and then click on the submission button.

\subsection{Managing Repository Data}

In the following figure, one other screenshot of the DECIDE Repository Manager is shown, (Fig. 10) when metadata are added to one of the previously uploaded and encrypted files.

\subsection{Performances and Usage Statistics}

As of the end of July 2012, more than 450 users belonging to about 120 organisations in more than 30 countries are registered in the Science Gateways developed by INFN Catania in the context of the projects mentioned in Section 2.2. The users registered in the DECIDE Science Gateway are in the order of 40 but this number is expected to rapidly increase in the near future as a training activity foreseen in the project has recently started and will further develop in the remaining months (the project will end at the end of February 2013).

Concerning performances, Fig. 11 shows the results of a stress-test of the Job Engine done on a single gLite [42] Workload Management System (WMS).

As it can be seen, up to 40,000 concurrent jobs can be managed at a constant rate of more than 10,000 jobs a hours. Above 30,000 concurrent jobs, the deterioration of the performances observer was due to the overload of the WMS and not to the Science Gateway. This very good result is a direct consequence of the use of the Thread Pools discussed in the previous sub-section.

Concerning web access speed and scalability, the DECIDE Science Gateway has been "measured" with Apache JMeter [43], a "de facto" standard in website performance evaluation, and no significant impact on the server load has been observed up to 600 users logging into the Science Gateway in 5 min (2 users a second) and submitting jobs at a global rate of 2 jobs a second.
Fig. 11 Results of a stress-test of the Job Engine on a gLite WMS
Job submission time (h)

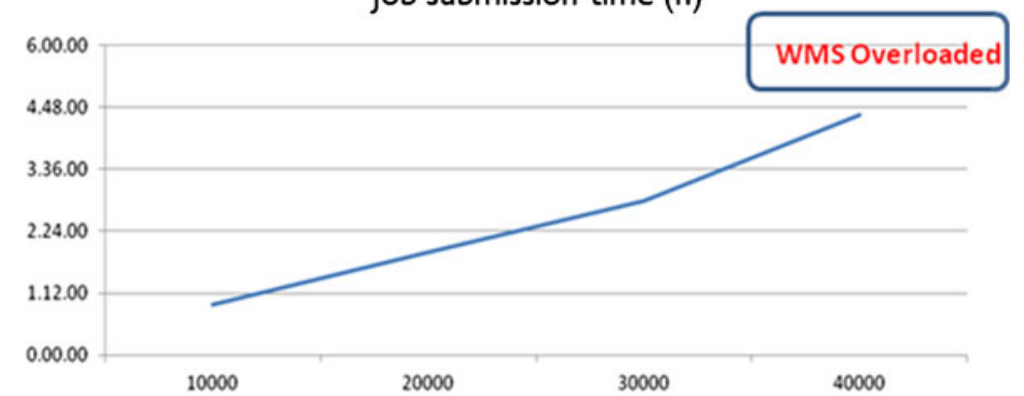

Time to submit 10,000 jobs (h)

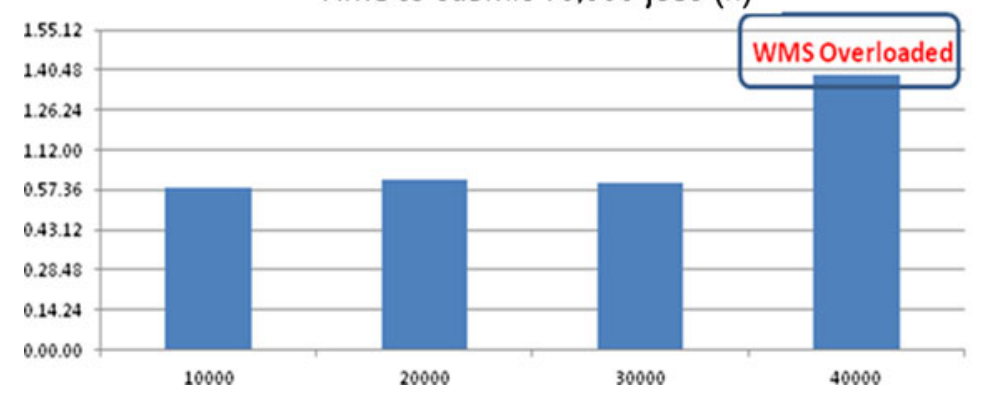


These figures are well beyond those expected in the project, at least in the forthcoming years.

\section{Summary and Conclusions}

The main goal of the DECIDE project is to uptake and exploit the e-Infrastructure paradigm in order to provide a dedicated production quality service for computer-aided diagnosis and research in the field of neurological diseases. DECIDE builds upon GËANT and EGI with the aim of fulfilling the specific needs of the neuroscientific and medical community. This will provide the community with new diagnostic and research tools and enable clinicians to tackle new challenges in their domain.

The services that have been realized by the DECIDE project are exposed to end users through a new type of Science Gateway based on the Liferay portlet container and on worldwide standards which makes use of a sophisticated authentication and authorization mechanism able to ease the access and use to Grid yet implementing a fine grained control on roles and corresponding privileges. The DECIDE Science Gateway allows also the creation and management of large distributed repositories of medical images with the possibility to encrypt the stored data.

The sustainability of DECIDE, at the level of infrastructure, is ensured by the fact that all sites which are part of the production service belong to organisations which are members of the National Grid Initiatives established in their countries. At the user level, different initiatives have been envisaged and already planned to reach long term sustainability. Examples are the training courses for the accurate use of the DECIDE services which will are being provided during the remaining months of the project.

Acknowledgements The research leading to these results was conducted as part of the DECIDE (Diagnostic Enhancement of Confidence by an International Distributed Environment) project. The authors acknowledge the financial support by the European Union FP7-ICT project DECIDE under contract $n^{\circ} .261593$. For further information, please visit www.eu-decide.eu.
Open Access This article is distributed under the terms of the Creative Commons Attribution License which permits any use, distribution, and reproduction in any medium, provided the original author(s) and source are credited.

\section{Glossary}

18F-DOPA fluorine 18 dihydroxyphenylalanine. 18F-FDG fluorine 18 fluoro-deoxy-glucose. 99TC-ECD technetium-99m-ethyl cysteinate diethylester.

AD Alzheimer Disease.

AMGA ARDA Metadata Grid Application.

API Application Programming Interface.

DECIDE Diagnostic Enhancement of Confidence by an International Distributed Environment EC FP7 project.

DS Discovery service.

EEG Electroencephalograpy.

EGI

GEANT Grid European Research Network.

gLite

GrIDP

HTTPS

IdF

IdP

INFN

JSAGA Java implementation of SAGA.

JSR

LCG

LDAP

Lightweight Middleware for Grid Computing.

Grid Identity Pool.

HyperText Transfer Protocol over Secure Socket Layer.

Identity Federation.

Identity Provider.

Italian National Institute of Nuclear Physics.

Java Specification Request.

LHC Computing Grid.

Lightweight Directory Access

Protocol.

LFC LCG File Catalogue.

LHC Large Hadron Collider.

LONI Laboratory of Neuro Imaging at UCLA.

MRI Magnetic Resonance Imaging.

NGI National Grid Initiative.

NREN National Research and Education

Network.

OGF Open Grid Forum.

PET Positron Emission Tomography.

REST REpresentional State Transfer. 


$\begin{array}{ll}\text { SAGA } & \text { Simple API for Grid Applications. } \\ \text { SAML } & \begin{array}{l}\text { Security Assertion Markup } \\ \text { Language. }\end{array} \\ \text { SP } & \text { Service Provider. } \\ \text { SSO } & \text { Single Sign On. } \\ \text { USB } & \text { Universal Serial Bus. } \\ \text { VO } & \text { Virtual Organization. } \\ \text { VOMS } & \text { Virtual Organisation Membership } \\ & \text { Service. } \\ \text { WMS } & \text { Workload Management System. }\end{array}$

\section{References}

1. DECIDE Project: http://www.eu-decide.eu. Accessed Oct 2012

2. GEANT Project: http://www.geant.net. Accessed Oct 2012

3. European Grid Infrastructure (EGI): http://www. egi.eu. Accessed Oct 2012

4. neuGRID Project: http://www.neugrid.eu. Accessed Oct 2012

5. Wilkins-Diehr, N.: Special Issue: Science Gateways Common Community Interfaces to Grid Resources. Concurrency Comput. Pract. Ex. 19(6), 743-749 (2007)

6. Wilkins-Diehr, N., Gannon, D., Klimeck, G., Oster, S., Pamidighantam, S.: TeraGrid Science gateways and their impact on science. IEEE Trans. Comput. 41(11), 32-41 (2008)

7. Castiglioni, I. et al.: A Grid-based SPM service (GriSPM) for SPECT and PET neurological studies. Eur. J. Nucl. Med. Mol. Imaging 36, 1193-1195 (2009)

8. Turkheimer, F.E., Aston, J.A.D., Asselin, M.C., Hinz, R.: Multi-resolution Bayesian regression in PET dynamic studies using wavelets. NeuroImage 32, 111-121 (2006)

9. Bose, S.K., Turkheimer, F.E., Howes, O.D., Mehta, M.A., Cunliffe, R., Stokes, P.R., Grasby, P.M.: The application of an artificial neural network to classification of schizophrenic patients and healthy controls using [18F] Fluorodopa PET imaging. Schizophr. Res. 106(2-3), 148-55 (2008)

10. Morra, J.H., Tu, Z., Apostolova, L.G., Green, A.E., Avedissian, C., Madsen, S.K., Parikshak, N., Hua, X., Toga, A.W., Jack, C.R. Jr., Weiner, M.W., Thompson, P.M., Alzheimer's Disease Neuroimaging Initiative: Validation of a fully automated 3D hippocampal segmentation method using subjects with Alzheimer's disease mild cognitive impairment, and elderly controls. Neuroimage 43(1), 59-68 (2008), Erratum in Neuroimage 44(4), 1439

11. Laboratory of Neuro Imaging of the University of California at Los Angeles: http://www.loni.ucla.edu. Accessed Oct 2012
12. Babiloni, F., Carducci, F., Cincotti, F., Del Gratta, C., Pizzella, V., Romani, G.L, Rossini, P.M., Tecchio, F., Babiloni, C.: Linear inverse source estimate of combined EEG and MEG data related to voluntary movements. Hum. Brain Mapp. 14, 197-209 (2001)

13. Babiloni, C. et al.: Directionality of EEG synchronization in Alzheimer's disease subjects. Neurobiol. Aging 30, 93-102 (2009)

14. Blinowska, K., Kus, R., Kaminski, M., Janiszewska, J.: Transmission of brain activity during cognitive task. Brain Topogr. 23, 205-213 (2010)

15. Wilkins-Diehr, N., Gannon, D., Klimeck, G., Oster, S., Pamidighantam, S.: TeraGrid Science gateways and their impact on science. IEEE Trans. Comput. 41(11), 32-41 (2008)

16. Wilkins-Diehr, N.: Special Issue: Science Gateways Common Community Interfaces to Grid Resources. Concurrency Comput. Pract. Ex. 19(6), 743-749 (2007)

17. Barbera, R. et al.: The GENIUS Grid Portal and robot certificates: a new tool for e-Science. BMC Bioinform. 10(Suppl. 6), S21 (2009)

18. Farkas, Z., Kacsuk, P.: P-GRADE Portal: a generic workflow system to support user communities. Future Generat. Comput. Syst. 27(5), 454 (2011)

19. Szejnfeld, D. et al.: Vine Toolkit - Towards portal based production solutions for scientific and engineering communities with grid-enabled resources support. Scalable Comput. Pract. Ex. 11(2), 161 (2010)

20. EGI VO Portal Policy: https://documents.egi.eu/public/ ShowDocument?docid=80. Accessed Oct 2012

21. EGI Grid Security Traceability and Logging Policy: https://documents.egi.eu/public/ShowDocument?docid=81. Accessed Oct 2012

22. The CHAIN project: http://www.chain-project.eu. Accessed Oct 2012

23. The EUMEDGRID-Support project: http://www. eumedgrid.eu. Accessed Oct 2012

24. The GISELA project: http://www.gisela-grid.eu. Accessed Oct 2012

25. The INDICATE project: http://www.indicate-project.eu. Accessed Oct 2012

26. The Liferay portal framework: http://www.liferay.com. Accessed Oct 2012

27. The JSR 286 standard: http://www.jcp.org/en/jsr/detail? $\mathrm{id}=286$. Accessed Oct 2012

28. The SAML standard: http://saml.xml.org. Accessed Oct 2012

29. The Shibboleth System: http://shibboleth.net/. Accessed Oct 2012

30. The GrIDP federation: http:/gridp.ct.infn.it. Accessed Oct 2012

31. The IDEM identity Federation: http://www.idem. garr.it. Accessed Oct 2012

32. The eduGAIN inter-federation: http://www.edugain.org. Accessed Oct 2012

33. La Rocca, G., Barbera, R., Ciaschini, V., Falzone, A., Monforte, S.: A new "lightweight" Crypto Library for supporting a new Advanced Grid Authentication Process with Smart Cards. In: Proceedings of Science (ISGC 2011 \& OGF 31), pp. 29 (2011) 
34. Calanducci, A. et al.: A Digital Library Management System for the Grid. In: Fourth International Workshop on Emerging Technologies for Next-generation GRID (ETNGRID 2007) at 16th IEEE International Workshops on Enabling Technologies: Infrastructures for Collaborative Enterprises (WETICE-2007), GET/INT Paris, France, 18-20 June 2007

35. The AMGA Metadata Catalogue: http://amga.web. ch/amga. Accessed Oct 2012

36. Scardaci, D., Scuderi, G.: A Secure Storage Service for the gLite Middleware, Proceedings of the Third International Symposium on Information Assurance and Security, pp. 261-266 (2007)

37. Scardaci, D.: Using Secure Storage Service inside the EELA-2 Infrastructures. In: Proceedings of the Second EELA-2 Conference, pp. 167-173 (2009)

38. Jha, S., Kaiser, H., Merzky, A., Weidner, O., et al:: Grid Interoperability at the Application
Level Using SAGA. In: Proceeding of IEEEInternational Conference on e-Science and Grid Computing, pp. 584-591. Bangalore, India, 10-13 December 2007

39. The SAGA OGF Standard Specification: http://www. gridforum.org/documents/GFD.90.pdf. Accessed Oct 2012

40. The JSAGA website: http://grid.in2p3.fr/jsaga/. Accessed Oct 2012

41. Rotondo, R., Fargetta, M., Barbera, R.: A Data Engine for Science Gateways: enabling easy data transfer and sharing. In: Proceedings of The International Symposium on Grids and Clouds (ISGC) 2012. PoS(ISGC 2012)039. http://pos.sissa.it/archive/conferences/153/ 039/ISGC\%202012_039.pdf

42. gLite middleware: http://glite.cern.ch. Accessed Oct 2012

43. Apache JMeter: http://jmeter.apache.org. Accessed Oct 2012 